\title{
Unfolding kinetic fragility in relaxor ferroelectrics
}

Julio C. Martínez-García, ${ }^{1, a)}$ Rafael Levit, ${ }^{2}$ Diego A. Ochoa, ${ }^{2}$ and José E. García ${ }^{2, a)}$

${ }^{1}$ Group of Nanomaterials and Microsystems, Department of Physics, Universitat Autònoma de Barcelona, Bellaterra 08193, Spain

${ }^{2}$ Department of Physics, Universitat Politècnica de Catalunya-BarcelonaTech, Barcelona 08034, Spain

\begin{abstract}
The fragility parameter is one of the most important material constants that is extensively used in glass science, playing a central role in the enhancement of the understanding the glass formation process of disordered systems. Although fragility has been widely used, this concept has never been precisely defined and evaluated in relaxor ferroelectrics. Here, we have filled up this scientific gap. Based on a generalized Vogel-Fulcher-Tammann equation, the fragility parameter is introduced for relaxor ferroelectrics. The new formulation has been quantitatively assessed by combining dielectric spectroscopy and pyroelectric measurements on canonical relaxors. A clear correlation between the fragility and a new local structural heterogeneity related order parameter elucidates new information about the ferroelectric order of relaxor ferroelectrics. A new pathway to disentangle relaxation phenomena in other relaxor ferroics could have opened.
\end{abstract}

\footnotetext{
a) Authors to whom correspondence should be addressed: jmartinezgarcia@gmail.com (J.C.M-G.) jose.eduardo.garcia@upc.edu (J.E.G.)
} 


\section{INTRODUCTION}

Understanding the glass formation process of disordered systems remains as one of the most attractive unresolved issues of condensed matter physics and material science engineering. ${ }^{1,2}$ The capability of these systems (e.g., molecular glasses) to retain the amorphous state (i.e., nonequilibrium) at low temperatures turns them into materials with no long-range translational order (periodicity) showing optimal properties in comparison with their crystalline counterparts. $^{3,4}$ Consequently, they have tremendous technological impact, ranging from pharmaceutical industry, geophysics and food science to modern electronics. ${ }^{5,6}$

Relaxor ferroelectrics (RF) are within this group of materials, which are considered to bear a revolutionary potential for a myriad of modern electronic applications because their unique properties such as ultrahigh strain, outstanding piezoelectric behavior, excellent electroacoustic response, and remarkable electro-optic properties..$^{7-9}$ Despite their doubtless technological impact, the explanation of their dynamic relaxation, however, are not yet well understood and, therefore, they are still a subject of intensive research.

The main intriguing feature of RF dynamics is their unexplained super-Arrhenius (SA) behavior, which manifests as a dramatic increase on the relaxation times in the linear response regime on cooling towards the material freezing state. ${ }^{10}$ This gives raise a thermally-activated process that strongly depend on the intrinsic properties (e.g., structure, dipolar interactions) of the material. As consequence, not all systems reach the glassy state in the same manner at same the cooling rate. They reach the vitreous state in different dynamic fashion (i.e., faster or slower). The temperature effect on the coupling degree of the relaxing entities (i.e., the polar nanoregions, PNRs, for RF) plays a dominant effect into the deviations degree from the Arrhenius behavior. ${ }^{11}$ It means that not only to clarify a consistent parameterization of the temperature dependence of the relaxation time is important but also how to accounts the deviations degree is crucial. 
Regarding of the searching of the optimal dynamic parameterization of the SA behavior in RF, decades of studies has engendered the prevailing conviction that it is possible via the VogelFulcher-Tammann (VFT) equation, which has been assumed as the key checkpoint for developing theories/models to describe the SA behavior of RF. Recently, however, it was demonstrated that VFT it is not always a proper approach for describing the dielectric relaxation in $\mathrm{RF}$, and a more general parameterization was formulated as: ${ }^{12}$

$$
\tau(T)=\tau_{0} \exp \left[\left(\frac{\Delta E_{0}}{k_{B}}\right)\left(\frac{T^{n-1}}{\left(T-T_{N}\right)^{n}}\right)\right]
$$

where a new local order parameter $n$ was defined. Note that VFT equation is recovered for the particular case of $n=1$, being $T_{N}$ the divergence temperature, known as freezing temperature for RF.

Once the optimal $\tau(T)$ parameterization is elucidated, the fragility concept is then used to quantitatively assess the Arrhenius deviations degree. This was introduced by Angell, providing a comprehensive understanding to the mechanism of the corresponding SA relaxation. A standardized representation to rescale the dynamics of glass forming materials was developed by Angell, ${ }^{13}$ which is known as Angell plot. It is based on the logarithm plot of a dynamical quantity (e.g. the relaxation times) versus a dimensionless temperature $u=T_{r} / T$, being $T_{r}$ a reference temperature usually associated with the glass transition temperature (Supplementary Information S1). Based on this plot, Angell classified glass materials as strong or fragile. Thus, materials exhibiting Arrhenius-type dependence follow a linear tendency and they are considered strong glass formers while those which deviate from such linear behavior are considered fragile glass formers. The deviation grade is assessed as the numerical value of the slope of the Angell representation at $u=1$, and it is termed as fragility parameter or kinetic fragility defined as:

$$
m=\left.\frac{d \log (\tau)}{d\left(\frac{T_{r}}{T}\right)}\right|_{T=T_{r}}
$$


The kinetic fragility serves as a quantitative valuable indicator for assessing the role of important thermodynamic quantities (e.g., specific heat, configurational entropy) into the dynamic relaxation. ${ }^{14-18}$ For instance, considering the Adam-Gibbs (AG) model, ${ }^{19}$ where the configurational entropy is linked to the relaxation time by the equation:

$$
\tau(T)=\tau_{0} \exp \left(\frac{\Delta E_{0}}{k T S_{c}(T)}\right)
$$

$\tau_{0}$ and $\Delta E_{0}$ being the relaxation time and activation energy at high temperatures, respectively, the fragility parameter can be written in a general manner (Supplementary Information S2) as:

$$
m=m_{0}\left[1+\left(\frac{d \ln S_{c}(T)}{d \ln T}\right)_{T=T_{r}}\right]
$$

where $m_{0}$ accounts the minimum value of fragility, which for the case of $T_{r}$ at $100 \mathrm{~s}$ gives rise $2-\log \tau_{0}$. By assessing the fragility, the role of fundamental effects such as dynamics, thermodynamic, order-disorder, the degree of cooperative motions, and the capability to form stable glasses, into the dynamics of glass formation may be elucidated ${ }^{20-25}$

The kinetic fragility has thus become an extraordinarily important indicator in glass science, which can be considered as a kind of "Gordian Knot constant" to improve the understanding of the glass formation process. However, it is extremely striking that fragility concept has been poorly used in RF although it has been deeply studied in molecular and metallic glasses. This is especially intriguing because RF and glass materials share two fundamental aspects: (i) both dynamics follow an SA behaviour and (ii) both systems fulfil AG considerations.

To the best of our knowledge, the work that goes the furthest attempt to correlate ferroelectric order and deviation degree of the SA behaviour in RF was published by Wang et $a l .{ }^{25}$ in 2014. However, three important issues of this work are not completely conclusive: (i) the results were obtained by rescaling the dynamics of RF at the same glass transition temperature defined for molecular glasses, but there is no experimental evidence showing that a glass transition occurs in RF at $100 \mathrm{~s}$, (ii) the authors invoked a dynamics parameterization but no expression for the kinetic fragility was derived, (iii) the tendency of fragility (strong or 
fragile) was evaluated by using a kinetic parameter $\left(K_{\mathrm{VFT}}\right)$, defined by Sastry, ${ }^{26}$ which arises from VFT fits, but VFT is not always the best parameterization to study the dynamics of RF.

Undoubtedly, there are important issues that deserves for its relevance within the RF field to be clarified. Here, we have bridged the gap between the ferroelectric order and SA behaviour of RF. Based on the generalized VFT equation, the fragility parameter has been introduced for RF. The new formulation has been quantitatively assessed by combining dielectric spectroscopy and pyroelectric measurements of two canonical RF. A clear correlation between the kinetic fragility and a new order parameter elucidates new insight information related to the ferroelectric order in RF. A new pathway to disentangle relaxation phenomena in other relaxor ferroics could have opened.

\section{FRAGILITY IN RELAXOR FERROELECTRICS}

In order to accurately estimate the fragility of a material, not only to elucidate the appropriate parameterization of $\tau(T)$ is important but also to clarify the appropriate reference temperature $T_{r}$ turns out to be essential. For molecular and metallic glasses, the dynamics is rescaled at the glass transition temperature $T_{g}$ as reference temperature, where the relaxation time $\tau$ reaches the value $100 \mathrm{~s}$ (i.e., $\tau\left(T_{g}\right)=100 \mathrm{~s}$ ). The assumption of $T_{r}=T_{g}$ for these materials have a solid foundation based on a set of experimental evidences (Supplementary Information S3). However, there is not experimental confirmation allowing $T_{g}$ to be taken as reference temperature for RF. Dissimilarity in some thermodynamic magnitude should be observed whether a pre-transitional thermal anomaly exists before RF reach the freezing temperature $T_{f}$. Precise specific heat measurements for $\mathrm{Pb}\left(\mathrm{Mg}_{1 / 3} \mathrm{Nb}_{2 / 3}\right) \mathrm{O}_{3}$ and $\mathrm{Pb}\left(\mathrm{Mg}_{1 / 3} \mathrm{Ta}_{2 / 3}\right) \mathrm{O}_{3}$ relaxors over a wide temperature range showed no anomalous signals at $T>T_{f} \cdot{ }^{27-29}$ Therefore, a new specific criteria should be adopted for RF in order to obtain a reference temperature $T_{r}>T_{f}$ from a common relaxation time, thereby appointing a characteristic value for the magnitude: 


$$
q=\log \tau\left(T_{r}\right)
$$

Note that $q=2$ for glass materials, but this value have to be lesser for RF. Figure 1 shows the temperature dependence of the relaxation time for $\left(\mathrm{Pb}_{0.91} \mathrm{La}_{0.09}\right)\left(\mathrm{Zr}_{0.35} \mathrm{Ti}_{0.65}\right) \mathrm{O}_{3}$ (hereafter labeled as $\mathrm{PLZT}$ ) and $0.9 \mathrm{PbMg}_{1 / 3} \mathrm{Nb}_{2 / 3} \mathrm{O}_{3}-0.1 \mathrm{PbTiO}_{3}$ (hereafter labeled as PMN-PT), two recognized canonical-prototype of RF. The PLZT data were obtained by using a precision LCR meter (Agilent E4980A) at a cooling rate of $0.2 \mathrm{~K} \mathrm{~min}^{-1}$ from $550 \mathrm{~K}$ to room temperature, whereas the PMN-PT data were taken from the Vielhand et al.${ }^{30}$ work (more information about the data and the data processing is given in Supplementary Information S4). Extrapolation of the data indicates that $\log \tau=0$ is obtained at temperature close to the freezing temperature (i.e., $T_{r} \approx T_{f}$ when $q=0$,). Hence, a value of $q<0$ should be adopted for RF. Taking into account the dynamics relaxation may be considered slow enough in these materials for frequencies $\sim 1 \mathrm{~Hz}$, we propose here to adopt $q=-1$ (i.e., $\tau(T)=10^{-1} \mathrm{~s}$ ) for RF (a detailed discussion about the $q$-value selection is given in Supplementary Information S3).

Once $q$ is stablished and, consequently, $T_{r}$ may be found, a generalized analytical equation for the fragility parameter can be obtained by substitute the proper $\tau(T)$ parameterization (Eq. (1)) in the Angell fragility definition (Eq. (2)) giving rise to (Supplementary Information S5):

$$
m=m_{0}\left[\frac{T_{r}-\left(T_{N}(1-n)\right)}{\left(T_{r}-T_{N}\right)^{(n+1)}}\right],
$$

being the minimum values of fragility

$$
m_{0}=\frac{\Delta E_{0}}{k_{B}}\left[\frac{1}{\ln (10) T_{r}^{(1-n)}\left(T_{r}-T_{N}\right)^{n}}\right]=\log \tau\left(T_{r}\right)-\log \tau_{0}
$$

The parameters $\tau_{0}$ and $\Delta \mathrm{E}_{0}$ define the relaxation time and activation energy at high temperatures, respectively, $n$ is a local order parameter and $T_{N}$ is the divergence (freezing) temperature. ${ }^{12,31,32}$ Noted that considering $T_{r}=T_{g}$ and $n=1$ the classical equation of the VFT-fragility is reproduced. 

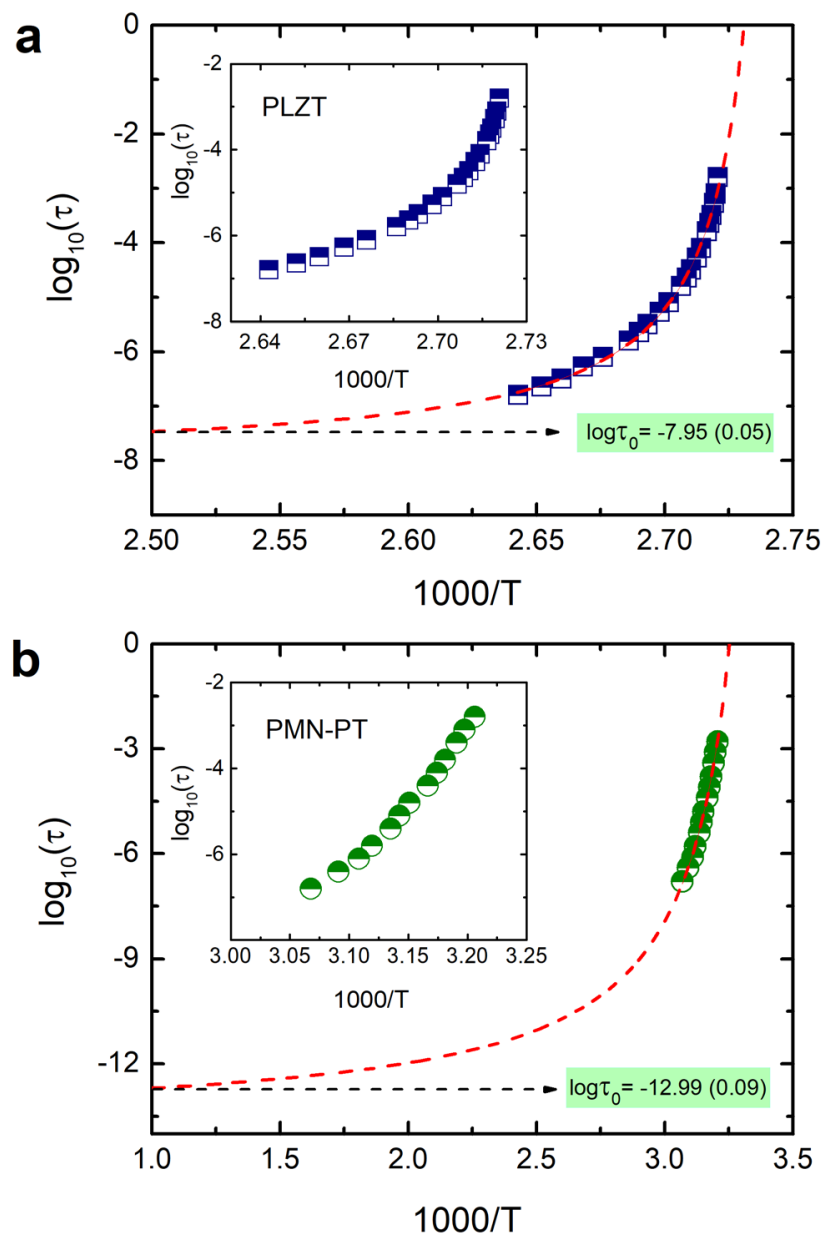

FIG. 1. Arrhenius plot. Logarithm of the relaxation time, calculated as the reciprocal of the measurement frequency, as a function of the inverse of the temperature corresponding to the maximum value of the real permittivity for (a), $\left(\mathrm{Pb}_{0.91} \mathrm{La}_{0.09}\right)\left(\mathrm{Zr}_{0.35} \mathrm{Ti}_{0.65}\right) \mathrm{O}_{3}$ (PLZT), and (b), $0.9 \mathrm{~Pb}\left(\mathrm{Mg}_{1 / 3} \mathrm{Nb}_{2 / 3}\right) \mathrm{O}_{3}-0.1 \mathrm{PbTiO}_{3}$ (PMN-PT). A clear super-Arrhenius behavior is manifested as a shift from the linear Arrhenius behavior. The classical VFT equation is used to fit the data in order to obtain the value of $\log \tau_{0}$. The result of the fitting is plotted in a wide range of temperatures. A zoom of the data representation is shown in the insets.

As shown in Eq. (6), the fragility constant is related to the local order parameter $n$, thereby revealing that $n$ plays a fundamental role for quantifying the Arrhenius deviation degree. The generalized VFT equation (Eq. (1)) can be written as:

$$
\log (\tau)=\log \left(\tau_{0}\right)+\left(\frac{E_{a}}{\ln 10 T_{r}}\right)\left[\frac{u}{\left(1-u \frac{T_{N}}{T_{r}}\right)^{n}}\right],
$$

by using the scaling temperature $u=T_{r} / T$. When $u=1$, Eq. (8) simplifies to:

$$
q=\log \left(\tau_{0}\right)+\left(\frac{E_{a}}{\ln 10}\right)\left[\frac{T_{r}^{n-1}}{\left(T_{r}-T_{N}\right)^{n}}\right]
$$

yielding 


$$
\left(T_{r}-T_{N}\right)^{n}=T_{r}^{n-1}\left[\frac{\left(\frac{E a}{\ln 10}\right)}{q-\log \left(\tau_{0}\right)}\right] .
$$

Hence, the reference temperature can be determined by solving Eq. (10) for a predefined $q$ value and a predetermined $n$ value. For the particular case of $n=1$ (i.e., classical VFT) and $q=2$ the reference temperature (i.e., the glass transition temperature) recovers the established formula used for glass materials. ${ }^{21,23}$

An Angell plot like representation can be obtained from Eq. (8) to explore the $n$ effect into the dynamics of RF in term of fragility. Fig. 2 shows a graphical solution of Eq. (8) for some values of the $n$-parameter taking an standardized value of the activation energy and computing $T_{r}$ for $q=-1$ (Supplementary Information S6). As may be observed, the value of the $n$ correlates with the dynamics properties of RF. Materials with small values of $n$ relax faster (smaller values of relaxation time) being more fragile than materials with higher $n$ values. As strong is the material as higher is their order parameter $n$.

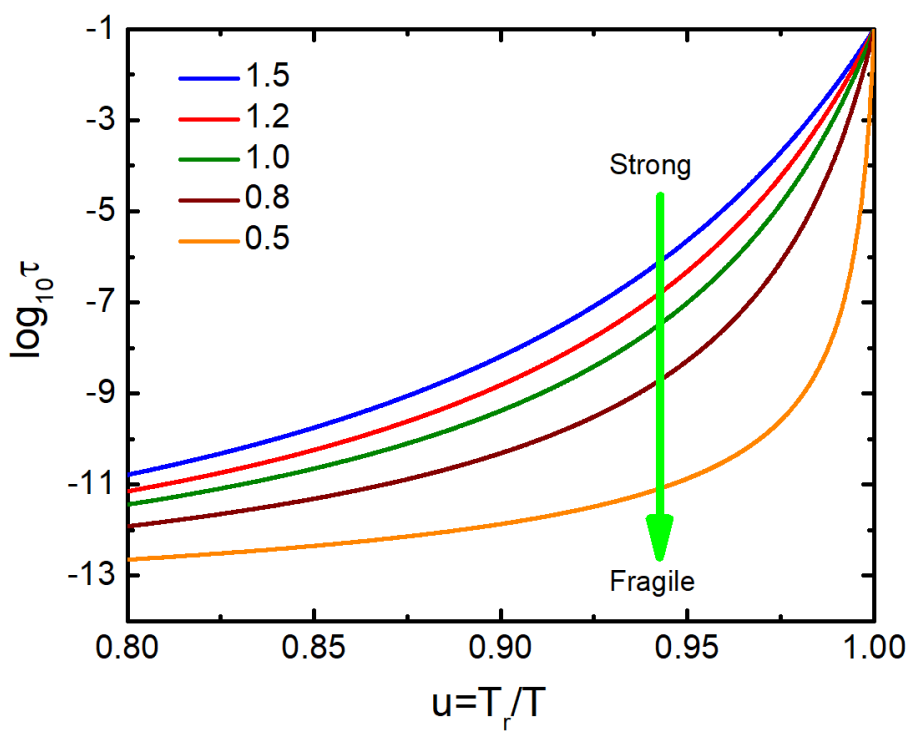

FIG. 2. Angell plot representation for relaxor ferroelectrics. Graphical representation of $\log \tau$ versus the dimensionless temperature $u$, for several values of the order parameter $n(1.5$, $1.2,1,0.8,0.5)$. A hypothetical freezing temperature of $290 \mathrm{~K}$ and a value of $E_{a} / \ln 10=250$ are used. A clear correlation between the value of $n$ and fragility is evidenced. A value of $q=$ -1 is chosen but any other $q$-value results the same behaviour. 


\section{A NEW REPRESENTATION FOR THE DYNAMICS DATA}

In order to disentangle the role of the local order parameter $n$ into the dynamic relaxation of RF a novel plot is now proposed. The generalized VFT equation (Eq. (1)) can be rewritten as follow:

$$
\log \left(\frac{\tau}{\tau_{0}}\right)=\left(\frac{E_{a}}{\ln 10}\right)\left[\frac{T^{n-1}}{\left(T-T_{N}\right)^{n}}\right]
$$

giving rise to:

$$
\left[\log \left(\frac{\tau}{\tau_{0}}\right)\right]^{-1}=A\left[\frac{\left(T-T_{N}\right)^{n}}{T^{n-1}}\right]
$$

being $A=\ln 10 / E_{a}$. The above transformation yields $\left[\log \left(\frac{\tau}{\tau_{0}}\right)\right]^{-1}$ versus temperature plot that serves as a valuable tool to estimate the order parameter $n$, the energy barrier parameter $A$ as well as the divergence (freezing) temperature $T_{N}$. The particular case classical VFT is obtained for $n=1$ turning out to be Eq. (12) in a linear function with a positive slope:

$$
\left[\log \left(\frac{\tau}{\tau_{0}}\right)\right]^{-1}=A T-\left(A T_{N}\right)
$$

defined in the temperature domain $T>T_{N}$. Undoubtedly, this novel plot becomes a relevant tool to test the validity of VFT equation, since any nonlinear behavior in this representation is directly related to a non-VFT behavior.

Figure 3 shows $\left[\log \left(\frac{\tau}{\tau_{0}}\right)\right]^{-1}$ versus T plot for both PMN-PT and PLZT. The PMN-PT data seem to exhibit a linear behavior while the PLZT data display a clear curvature, reinforcing that classical VFT equation is not always the proper equation to describe the dynamic of RF systems. The freezing temperature of theses RF systems can be assessed from pyroelectric measurement (Supplementary Information S7) while the value of $\tau_{0}$ can be determined from the dielectric relaxation data (Supplementary Information S8). Thus, the parameter $n$ can be easily obtained by fitting the data. The results are plotted in Fig. 3. As can be observed, a value of $n=0.98$ is obtained for PMN-PT, very close to $n=1$, in concordance with previous results by Viehland 
et al. ${ }^{30}$ However a value of $n=0.54$ is found for PLZT, strengthening that the generalized VFT equation (Eq. (1)) should be taking into account for RF.
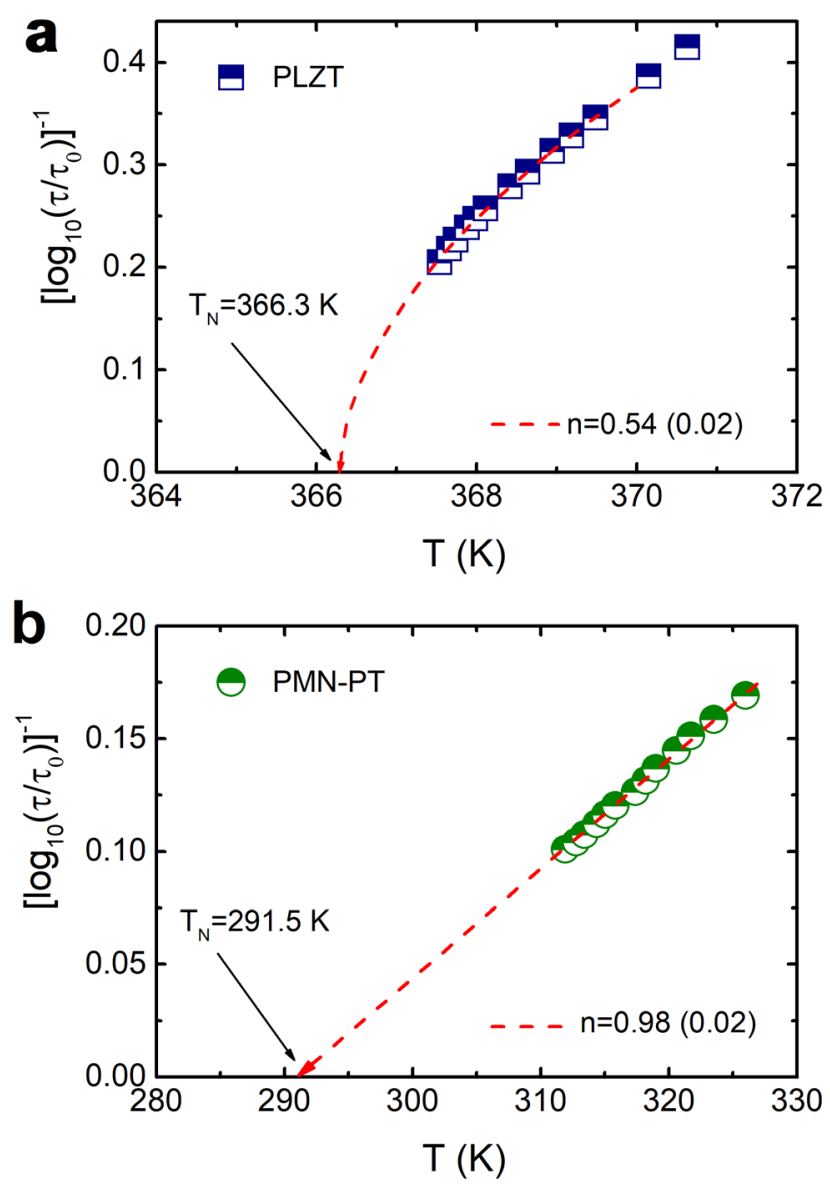

FIG. 3. Novel representation of the relaxation data. The inverse of $\log _{10}\left(\tau / \tau_{0}\right)$ is plotted as a function of temperature for (a) PLZT and (b) PMN-PT. The values of the freezing (divergence) temperature are previously obtained from pyroelectric measurement in order to fit the data to Eq. (12). The obtained values of $n$-parameters are shown.

Once all the parameters have been determined, the kinetic fragility can be computed from Eq. (6) and (9). Furthermore, the impact of the $q$ value can also be assessed, the results being shown in Fig. 4. As may be observed, both the fragility parameter and the reference temperature notably depends on the $q$ value but the tendency of the material to be stronger or more fragile turn out to be independent of $q$. The dependence with $q$ of both $T_{r}$ and $m$ is more linear (less curved) for PMN-PT than for PLZT, which is conditioned by the value of the difference $\Delta T$ between the reference and freezing temperatures. The value of $\Delta T$ is higher for PMN-PT $(\sim 19$ $\mathrm{K})$ than for PLZT $(\sim 3 \mathrm{~K})$ and it is related to the fragility parameter resulting the PLZT more 
fragile $(m=486)$ than the PMN-PT $(m=186)$. It is important to point out that the fragility values for RF should not be compared with the values established for conventional glasses. In the case of conventional glasses, the fragility is experimentally measured close to the glass transition point $\left(\tau\left(T_{r}\right)=100 \mathrm{~s}\right)$, but this concept cannot be extrapolated to RF as discussed before. We are establishing here a criterion to calculate the fragility for RF by taking the value for $q=-1$ which could be a future reference criterion within the relaxor community to compare fragility values for RF and to evaluate the curvature degree of the SA behavior of these materials.
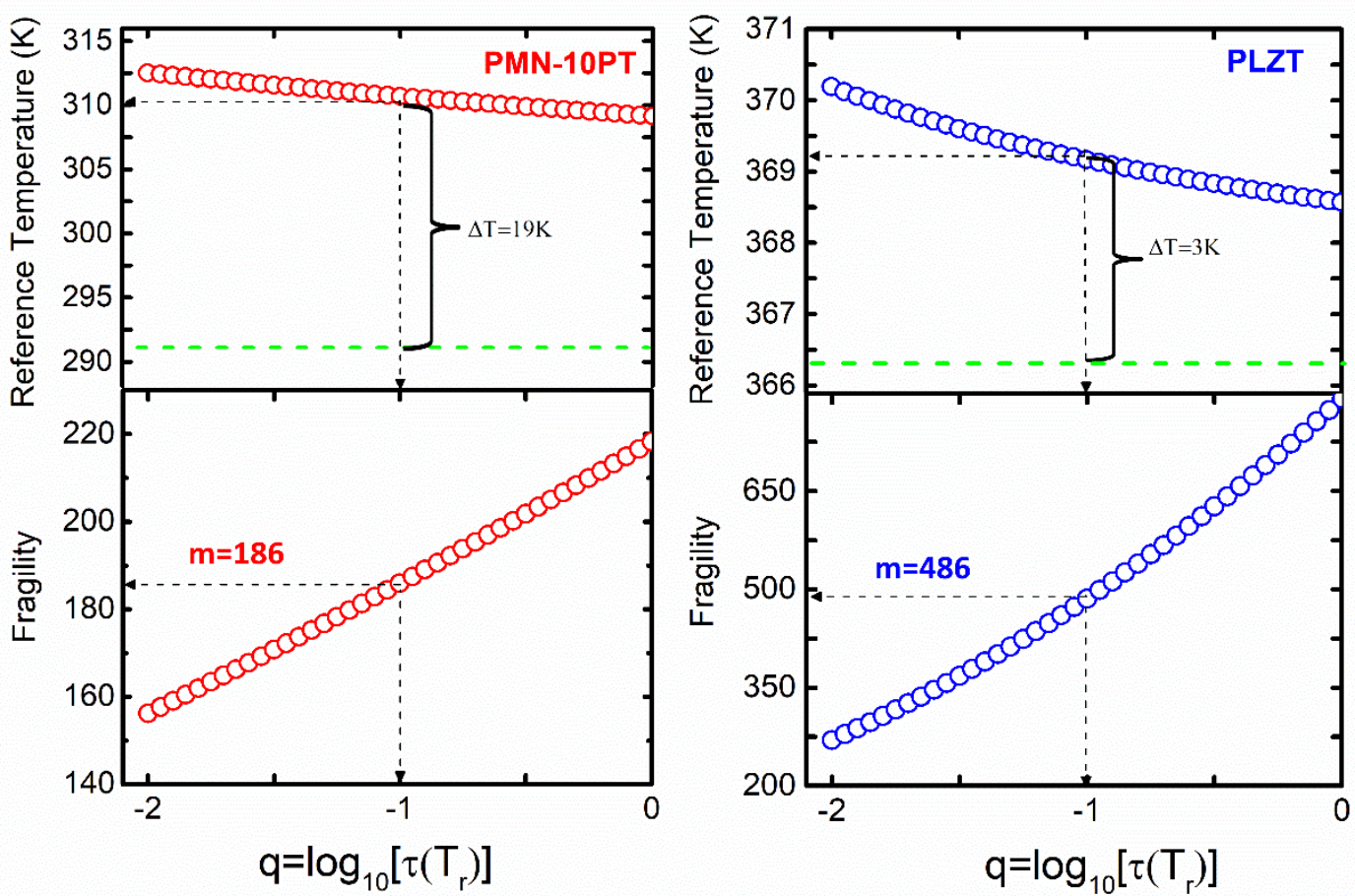

FIG. 4. Fragility and reference temperature. Evaluation of the $q$-impact into the fragility parameter and the reference temperature for both PMN-PT and PLZT. Freezing temperatures are indicated by a green dash line. The values of the fragility parameter is explicitly shown for $q=-1$

\section{DYNAMICS AND FERROELECTRIC ORDER}

The ferroelectric order of RF is usually linked to the correlation length, $\xi$, which is a direct measure of the length scale of PNRs. Neutron scattering and nuclear magnetic resonance techniques have shown $\xi$ increases on cooling and changes remarkably around certain 
temperature $\left(T_{f}\right)$ below which a saturation takes place suggesting a possible percolation-type transition into a frozen relaxor state where the appearance of a frozen polar glass phase arises. ${ }^{33,34}$ On cooling, the dipoles find each other forming the mentioned PNRs. As the material cools down these regions grow larger becoming slow-moving increasingly difficult for them to respond to external stimuli such as electric fields. This fact implies that the dramatic increase of relaxation time (the SA behavior) observed in RF takes place as a direct consequence of a remarkable increase of the correlation length of their PNRs. ${ }^{35-37}$ If $\tau\left(T_{f}\right) \rightarrow \infty$, then it must be accompanied by a divergence of the correlation length; i.e., $\xi\left(T_{f}\right) \rightarrow \infty$.

Considering the arguments of the AG theory, $\xi$ can be directly correlated with the temperature evolution of the configurational entropy. On cooling towards $T_{f}$, the configurational entropy of the system diminishes as the size of the PNRs grows leading to an increase in the relaxation time. Based on that, we can claim that the growth of the correlation length-scale in RF takes place as a direct consequence of the decrease of the configurational entropy. In principle, a simple inverse relation of proportionality between configurational entropy $S c$ and the correlation length, $S c(T) \propto 1 / \xi(T)$, could be invoked. However, two important issues should be taking into account: (i) although AG theory provides a precise link connection between $S c$ and $\tau$, it does not provide information about the temperature evolutions of the absolute size of PNRs and (ii) the dimensionality should also be considered. Bringing the arguments of the random first order theory (RFOT), ${ }^{38-40}$ which generalizes the AG configurational entropy equation, ${ }^{41,42}$ the SA behavior can be explained in terms of a dramatic increase of the cavity size of relaxing entities on cooling, quantified by the correlation length as $\operatorname{Sc}(T) \propto(1 / \xi(T))^{d-\theta}$, being $\theta$ an interface exponent and $d$ the dimensionality. By using finite-dimensional surface fluctuations, Krikpatrick et al. ${ }^{38}$ found that $\theta=d / 2$ giving rise the following general relationship:

$$
S_{C}(T) \propto\left(\frac{1}{\xi(T)}\right)^{\frac{d}{2}} .
$$


The above arguments can be straightforward linked to the dynamic relaxation of RF. Based on the RFOT, the PNRs can also be considered as a cavity of relaxing entities whose size dramatically increases on cooling. Therefore, Equation (14) can provide a quantitative link connection between the correlation length and configurational entropy of RF. Considering the generalized entropy equation of RF: ${ }^{12}$

$$
S_{c}(T)=S_{0}\left[\left(1-\frac{T_{N}}{T}\right)^{n}\right]
$$

where $S_{0}$ is the configurational entropy at high temperatures, and substituting it in Eq. (14) the following relationship is obtained:

$$
\xi(T) \propto \frac{1}{\left(1-\frac{T_{N}}{T}\right)^{\frac{2 n}{d}}} .
$$

As shown in Eq. (16) the $n$-parameter plays an important role to quantitative evaluate the growth of the correlation length of RF. In order to evaluate the effect of $n$, Eq. (16) is rewritten by using the scaling temperature $u=T_{r} / T$ resulting:

$$
\xi(T) \propto \frac{1}{\left(1-\frac{T_{N}}{T_{r}} u\right)^{\frac{2 n}{d}}} .
$$

Normalizing by $\xi\left(T_{r}\right)$ we obtain:

$$
\frac{\xi(T)}{\xi\left(T_{r}\right)}=\left(\frac{T_{r}-T_{N}}{T_{r}-u T_{N}}\right)^{\frac{2 n}{d}}
$$

Following the same procedure, the configurational entropy can also be rescaled by $S_{c}\left(T_{r}\right)$ yielding:

$$
\frac{S_{C}(T)}{S_{c}\left(T_{r}\right)}=\left(\frac{T_{r}-u T_{N}}{T_{r}-T_{N}}\right)^{n}
$$

Figure 5(a) shows the Angell plot like representation of the relaxation data for the studied RF systems. Additionally, scaling plots of Eq. (18) and (19) are also shown as insets of the figure. As may be observed, PLZT exhibits a more pronounced curvature than PMN-PT. 
This can be explained because the A-site vacancies generated by the substitution of $\mathrm{La}^{3+}$ for $\mathrm{Pb}^{2+}$ in PLZT result in weaker coupling dipolar interaction among PNRs, giving rise to a lower values of the relaxation time (i.e., faster relaxation dynamic) manifested by higher values of configurationally entropy and lower values of correlation length, thereby denoting lower activation energy to relax (fragile material). Hence, PLZT exhibits a more pronounced curvature than PMN-PT, indicating PLZT is more fragile as was shown above (Fig. 4). Furthermore, PLZT follow the direction of the electric field easier and, therefore, lower values of $n$ imply higher ferroelectric order ascribed to lower coupling interactions. All the discussion is schematically summarized in Fig. 5(b).

\section{a}

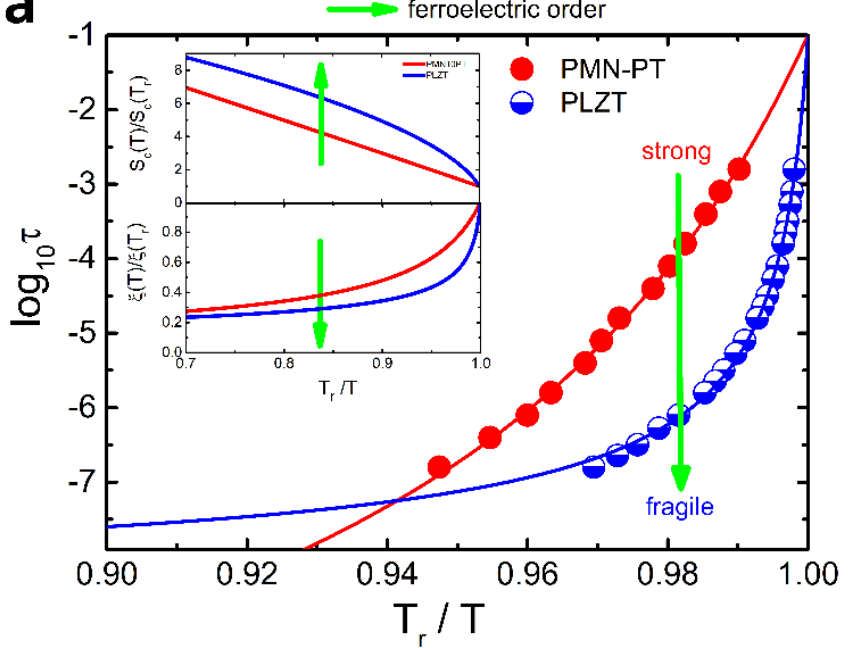

b

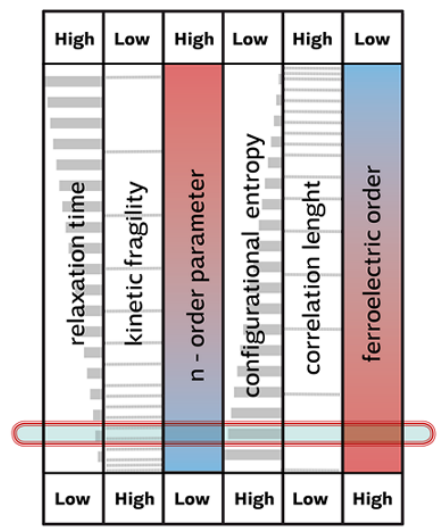

FIG. 5. Scaling plots and magnitudes correlation. (a) Angell plot representation for the tested materials, taking $q=-1$ to calculate the values of $T_{r}$. Solid lines indicate the data fitting by using the generalized VFT equation. The fitting was performed for the low temperature dynamic data but it is plotted for a broader temperature range to guide the eyes. The inset shows the normalized configurational entropy and correlation length for both materials. (b) Schematic representation of the correlation between several dynamics magnitudes.

Different dynamics behavior are found related to the value of the $n$-parameter, which in turn depend on the compositional heterogeneity order of the tested RF. Higher value of $n$ is obtained for a stoichiometric solid solution (PMN-PT) where the chemical inhomogeneities are fixedly randomly distributed, while a non-stoichiometric solid solution (PLZT) exhibits lower 
value of $n$. Thus, $n$ becomes a parameter directly connected with the dynamics and ferroelectric order and associated with the configurational entropy and correlation length of PNRs.

\section{CONCLUSION}

The fragility concept is developed for relaxor ferroelectrics. Our results reveal that the interacting relaxing entities (PNRs) of PMN-PT require more efforts to follow the applied electric field, which is manifested with a higher values of the relaxation times (i.e., slow relaxation dynamics) ascribed to less pronounced curvature on the Angell plot giving rise lower values of fragility. Higher values of correlation length and lower values of configurationally entropy are obtained, involving higher activation energy to relax (i.e., strong material). These facts indicate that higher values of $n$ entail lower ferroelectric order ascribed to stronger coupling interactions. Summarizing, like in super-cooled liquids, where the dynamic metric (fragility) is directly related with the degree of their dipolar interactions (i.e., hydrogen bonding), the dynamic metric of RF can also be directly connected with the degree of the coupling interaction between PNRs. Our results elucidate that fragility of the material is intrinsically correlated with the ferroelectric order, where the order parameter $n$ plays an important role. Undoubtedly, the obtained results shed a new light to understand the dynamic relaxation of RF where the here implemented approach may open a new pathway to disentangle relaxation phenomena in other relaxor ferroics.

\section{SUPPLEMENTARY MATERIAL}

See supplementary material for: (i) the complete description of the Angel plot; (ii) the demonstration of the fragility equation based on the Adam-Gibb model; (iii) the discussion of the $q$-value selection; (iv) more information about the data and the data processing; (v) the demonstration of the generalized fragility equation; (vi) a description about the interrelation 
between the fragility parameter $m$ and the local order parameter $n$; (vii) the pyroelectric measurements; (viii) the estimation of $\tau_{0}$ from the dielectric relaxation data.

\section{ACKNOWLEDGEMENTS}

This work was partially supported by the project PGC2018-099158-B-I00 of the Spanish Government (Agencia Estatal de Investigación) and by the Catalan Government within the Beatriu de Pinos program, grant number NB615798 (J.C.M-G.).

\section{DATA AVAILABILITY}

The data that supports the findings of this study are available within the article and its supplementary material.

\section{REFERENCES}

1 P. G. Debenedetti and F. H. Stillinger, Nature 410, 259 (2001).

2 D. Kennedy, Science 309, 83 (2005).

3 J. J. Kruzic, Adv. Eng. Mater. 18, 1308 (2016).

4 E. Axinte, Mater. Des. 32, 1717 (2011).

5 N. R. Jadhav, V. L. Gaikwad, K. J. Nair, and H. M. Kadam, Asian J. Pharm. 3, 82 (2009).

6 Y. H. Roos, Ann. Rev. Food Sci. Technol. 1, 469, (2010).

7 F. Li, S. Zhang, T. Yang, Z. Xu, N. Zhang, G. Liu, J. Wang, J. Wang, Z. Cheng, Z.-G. Ye, J. Luo, T. R. Shrout, and L.-Q. Chen, Nat. Commun. 7, 13807 (2016).

8 S. Zhang, F. Li, X. Jiang, J. Kim, J. Luo, and X. Geng, Prog. Mater. Sci. 68, 1 (2015).

9 E. Sun and W. Cao, Prog. Mater. Sci. 65, 124 (2014).

10 A. A. Bokov and Z.-G. Ye, J. Mater. Sci. 41, 31 (2006).

11 W. Kleemann, J. Mater. Sci. 41, 129 (2006).

12 R. Levit, J. C. Martinez-Garcia, D. A. Ochoa, and J. E. Garcia, Sci. Rep. 9, 12390 (2019).

13 C. A. Angell, J. Non-Cryst. Solids 131-133, 13 (1991).

${ }^{14}$ L-M. Wang and C. A. Angell, J. Chem. Phys. 118, 10353 (2003).

15 L-M. Wang, C. A. Angell, and R. Richert, J. Chem. Phys. 125, 074505 (2006).

16 V. Lubchenko and P. G. Wolynes, J. Chem. Phys. 119, 9088 (2003). 
17 J. D. Stevenson and P. G. Wolynes, J. Phys. Chem. B 109, 15093 (2005).

18 P. K. Gupta and J. C. Mauro, Phys. Rev. E 78, 062501 (2008).

19 G. Adam and J. H. Gibbs, J. Chem. Phys. 43, 139 (1965).

${ }^{20}$ R. Böhmer, J. Non-Cryst. Solids 172-174, 628 (1994).

21 R. Böhmer, K. L. Ngai, C. A. Angell, and J. D. Plazek, J. Chem. Phys. 99, 4201 (1993).

22 U. Mohanty, N. Craig, and J. T. Fourkas, J. Chem. Phys. 114, 10577 (2001).

${ }^{23}$ Z. Chen and R. Richert, J. Chem. Phys. 135, 124515 (2011).

24 A. Sepúlveda, M. Tylinski, A. Guiseppi-Elie, R. Richert, and M. D. Ediger, Phys. Rev. Lett. 113, 045901 (2014).

25 Y-Z. Wang, L. Chen, H-Y. Wang, X. F. Zhang, J. Fu, X-M. Xiong, and J-X. Zhang, J. Appl. Phys. 115, 054106 (2014).

26 S. Sastry, Nature 409, 164 (2001).

27 Y. Moriya, H. Kawaji, T. Tojo, and T. Atake, Phys. Rev. Lett. 90, 205901 (2003).

28 W. Kleemann, J. Dec, V. V. Shvartsman, Z. Kutnjak, and T. Braun, Phys. Rev. Lett. 97, 06702 (2006).

${ }^{29}$ M. Tachibana, K. Sasame, H. Kawaji, T. Atake, and E. Takayama-Muromachi, Phys. Rev. B 80, 094115 (2009).

30 D. Viehland, S. J. Jang, L. E. Cross, and M. Wuttig, J. Appl. Phys. 68, 2916 (1990).

31 J. C. Martinez-Garcia, S. J. Rzoska, A. Drozd-Rzoska, and J. Martinez-Garcia, Nat. Commun. 4, 1823 (2013).

32 R. Levit, D. A. Ochoa, J. C. Martinez-Garcia, and J. E. Garcia, New J. Phys. 19, 113013 (2017).

33 G. Xu, G. Shirane, J. R. D. Copley, and P. M. Gehring, Phys. Rev. B 69, 064112 (2004).

34 I.-K. Jeong, T. W. Darling, J. K. Lee, Th. Proffen, R. H. Heffner, J. S. Park, K. S. Hong, W. Dmowski, and T. Egami, Phys. Rev. Lett. 94, 147602 (2005).

35 R. Pirc and R. Blinc, Ferroelectrics 379, 30 (2009).

${ }^{36}$ R. A. Cowley, S. N. Gvasaliya, S. G. Lushnikov, B. Roessli, and G. M. Rotaru, Adv. Phys. 60, 229 (2011).

37 R. Pirc and R. Blinc, Phys. Rev. B 76, 020101R (2007).

38 T. R. Kirkpatrick, D. Thirumalai, and P. G. Wolynes, Phys. Rev. A 40, 1045 (1989).

39 J.-P. Bouchaud and G. Biroli, J. Chem. Phys. 121, 7347 (2004).

40 V. Lubchenko and P. G. Wolynes, Annu. Rev. Phys. Chem. 58, 235 (2007).

41 M. Ozawa, C. Scalliet, A. Ninarello, and L. Berthier, J. Chem. Phys. 151, 084504 (2019). 
This is the post-print (i.e. final draft post-refereeing) of the publication.

The final publication is available at AIP Publishing via http://dx.doi.org/10.1063/5.0067913

${ }^{42}$ L. Berthier, P. Charbonneau, A. Ninarello, M. Ozawa, and S. Yaida, Nat. Commun. 10, 1508 (2019). 